\title{
PULSE PROPAGATION IN DISCRETE SYSTEMS OF COUPLED EXCITABLE CELLS*
}

\author{
A. CARPIO $^{\dagger}$ AND L. L. BONILLA ${ }^{\ddagger}$
}

\begin{abstract}
Propagation of pulses in myelinated fibers may be described by appropriate solutions of spatially discrete FitzHugh-Nagumo systems. In these systems, propagation failure may occur if either the coupling between nodes is not strong enough or the recovery is too fast. We give an asymptotic construction of pulses for spatially discrete FitzHugh-Nagumo systems, which agrees well with numerical simulations, and discuss the evolution of initial data into pulses and pulse generation at a boundary. Formulas for the speed and length of pulses are also obtained.
\end{abstract}

Key words. discrete reaction-diffusion equations, traveling wave pulses, propagation failure, spatially discrete FitzHugh-Nagumo system

AMS subject classifications. 34E15, 92C30

PII. S0036139901391732

1. Introduction. Effects of spatial discreteness are important in many physical and biological systems comprising interacting smaller components such as atoms, quantum wells, cells, etc. Examples include the motion of dislocations [13], crystal growth and interface motion in crystalline materials [6], the motion of domain walls in semiconductor superlattices [4, 8], sliding of charge density waves [14], and pulse propagation through myelinated nerves [2]. The mathematical study of spatially discrete models is challenging because of special and poorly understood phenomena occurring in them that are absent if the continuum limit of these models is taken. Paramount among these phenomena is the pinning or propagation failure of wave fronts in spatially discrete equations. Physically, the pinning of wave fronts is related to the existence of Peierls stresses in continuum mechanics [17], relocation of electric field domains [1] and self-sustained oscillations of the current in semiconductor superlattices [18, 4], electric current due to the sliding of charge density waves [14], saltatory propagation of impulses in myelinated fibers and its failure [2], etc.

Mathematical understanding of the propagation failure of wave fronts in spatially discrete equations experienced significant progress after a paper by Keener [20]. In [20], Keener used comparison principles to characterize the pinning of wave fronts and their motion for spatially discrete reaction-diffusion equations of the form

$$
u_{n, t}=d\left(u_{n+1}-2 u_{n}+u_{n-1}\right)+f\left(u_{n}\right)
$$

where $f$ is a bistable source term and $d$ measures the strength of the coupling. Models described by (1.1) include the spatially discrete Nagumo equation for nerve conduction

${ }^{*}$ Received by the editors November 26, 2001; accepted for publication (in revised form) May 30, 2002; published electronically December 11, 2002. This research was supported by the DGES grant PB98-0142-C04-01, by the MCyT grant BFM2002-04127-C02, by the Third Regional Research Program of the Autonomous Region of Madrid (Strategic Groups Action), and by the European Union under grant RTN2-2001-00349.

http://www.siam.org/journals/siap/63-2/39173.html

${ }^{\dagger}$ Departamento de Matemática Aplicada, Universidad Complutense de Madrid, 28040 Madrid, Spain (carpio@mat.ucm.es).

¥Departamento de Matemáticas, Escuela Politécnica Superior, Universidad Carlos III de Madrid, Avenida de la Universidad 30, 28911 Leganés, Spain, and Unidad Asociada al Instituto de Ciencia de Materiales de Madrid (CSIC), 28049 Cantoblanco, Spain, (bonilla@ing.uc3m.es). 
$[12,26]$ or crystal growth [6], and the Frenkel-Kontorova model for motion of dislocations [13]. More recently, a number of results on the existence of traveling wave fronts $u_{n}(t)=v(n-c t)$ with smooth profiles $v$ have been established [31, 24, 10]. These papers do not precisely characterize the propagation failure of a wave front for critical values of the control parameters. For a piecewise linear source function, an explicit description has been given by Fáth, extensively using the properties of special functions [11]. In the general case, we have found that propagation failure can be explained as a loss of continuity of the wave front profile in critical values of the control parameter [7]. Furthermore, the smoothness of the wave front profile just before the propagation failure occurs can be exploited to obtain an analytical description of wave fronts and their speed near critical parameter values [7,9]. This theory has been extended to spatially discrete reaction-diffusion-convection equations describing dynamics of domain walls in semiconductor superlattices [8].

These advances in the mathematical understanding of propagation phenomena have occurred for spatially discrete scalar reaction-diffusion equations. Similar phenomena occur in models of calcium release at discrete sites [22]. The latter consist of scalar reaction-diffusion equations with spatially inhomogeneous source terms that are close to $f(u)$ times a series of delta functions centered at spatially periodic sites. Comparatively little progress has been made in understanding wave propagation and failure in spatially discrete systems. Anderson and Sleeman [2] have extended Keener's techniques to discrete reaction-diffusion systems modelled by the FitzHugh-Nagumo (FHN) dynamics [12, 26]. Hastings and Chen [16] have proved the existence of pulse traveling waves for a myelinated nerve model with a Morris-Lecar type of dynamics. They also comment on the difficulties of extending their results to the FHN system. An attempt to understand the mechanisms of propagation failure in the FHN system has been carried out by Booth and Erneux [5]. They consider slow recovery and very special limiting (small) values of the parameters characterizing the bistable source and the spatial diffusivity in the FHN system. Furthermore, they also impose particular boundary and initial conditions. With these restrictions, they could study how a specific disturbance localized in one cell propagated to neighboring ones until the resulting front failed to propagate. No construction of pulses or formulas for their velocity were given.

In this paper, we asymptotically construct pulse solutions of the spatially discrete FHN system describing nerve conduction through myelinated fibers. We also discuss how the pulses may fail to propagate. Our ideas could be extended to spatially discrete systems whose cell dynamics contain widely separated time scales corresponding to fast excitation and slow recovery variables. Among these systems, let us cite models for bursting behavior in pancreatic $\beta$ cells [30] or the much more difficult case of front propagation in voltage-biased semiconductor superlattices [4]. In the latter, a separation of time scales exists, but it is not obviously included as a small parameter in the equations. In our presentation, we have chosen the FHN dynamics for its simplicity. This model has been widely used to understand issues that are obscured by technical complications in more realistic models of nerve conduction. We consider the following system of dimensionless equations:

$$
\begin{aligned}
\epsilon \frac{d u_{n}}{d t} & =d\left(u_{n+1}-2 u_{n}+u_{n-1}\right)+A u_{n}\left(2-u_{n}\right)\left(u_{n}-a\right)-v_{n}, \\
\frac{d v_{n}}{d t} & =u_{n}-B v_{n}
\end{aligned}
$$

$n=0, \pm 1, \ldots$ Here $u_{n}$ and $v_{n}$ are the membrane potential and the recovery variable 


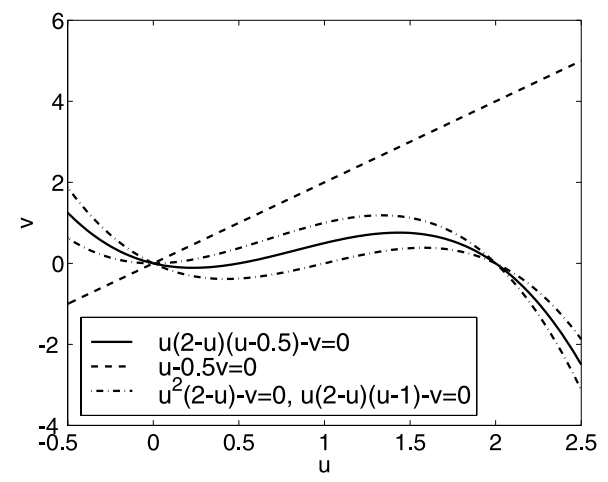

FIG. 1.1. Nullclines for the space-independent FHN model with different values of a.

(which acts as an outward ion current) at the $n$th excitable membrane site (node of Ranvier). The cubic source term is an ionic current, and the discrete diffusive term is proportional to the difference in internodal currents through a given site. The constants $A$ and $B$ are selected so that the source terms in the FHN system are $O(1)$ for $u_{n}$ and $v_{n}$ of order 1 , that the only stationary uniform solution is $u_{n}=0=v_{n}$, and that the FHN system has excitable dynamics $(A=1, B=0.5$ is a good choice; see Figure 1.1). The constant $\epsilon>0$ is the ratio between the characteristic time scales of both variables. We assume $\epsilon \ll 1$, that is, fast excitation and slow recovery. A dimensional version of (1.2) and (1.3) was derived in the appendix of [3] from an equivalent-circuit model of myelinated nerves. For background on similar models, see $[28,29,21,25]$.

We shall study pulse propagation in the spatially discrete FHN system (1.2) and (1.3) by asymptotic methods. At first sight, such a task is hopeless: asymptotic methods require a degree of smoothness at appropriate time or length scales, and the spatial variable $n$ in these systems is discrete. However, we can use the separation between time scales in the FHN system to show that a pulse is made out of two "sharp" wave fronts separating regions of slow spatial variation. Wave fronts are smooth solutions of the continuous variable $z=n-c t / \epsilon$, and perturbative arguments apply straightforwardly to them. Thus the theory of wave front propagation for spatially discrete scalar reaction-diffusion equations plays an important role in our construction of pulses.

Let $U_{1}<U_{2}<U_{3}$ denote the three zeros of the cubic nonlinearity $f(u)$ in (1.1). $U_{1}$ and $U_{3}$ are stable solutions for $d=0$. A wave front is a solution of (1.1) with a smooth profile $u_{n}(t)=u(n-c t)$ moving at a speed $c$ such that $u(\mp \infty)=U_{1}$ and $u( \pm \infty)=U_{3}$. If $f(u)$ is odd about $u=U_{2}$ and $d$ is sufficiently small, a stationary solution of (1.1) exists and therefore no wave fronts can propagate (see [10]). As the source term departs from this symmetric form, front propagation is made easier. In [7], we selected $d=1$ and $f(u)=F-A g(u)$, where $g(u)$ is odd about its middle zero and $F$ is an external force that quantifies departure from symmetry. Notice that we can obtain (1.1) with $d=1 / A$ and $f=(F / A)-g(u)$ after rescaling time. We found that wave fronts propagate for $|F|>F_{c}$, where $F_{c}>0$ depends on $A$ and the specific $g(u)$ that we adopt. Equivalently, we could set $f(u)=-u(u-a)(u-2)$ and use $a-1$ as a control parameter instead of $F$. After the "external force" $a$ surpasses a critical value sufficiently far from the symmetry point $a=1$, stationary fronts may cease to exist and propagating wave fronts may appear. See Figure 1.2(a). Notice that the limiting 
(a)

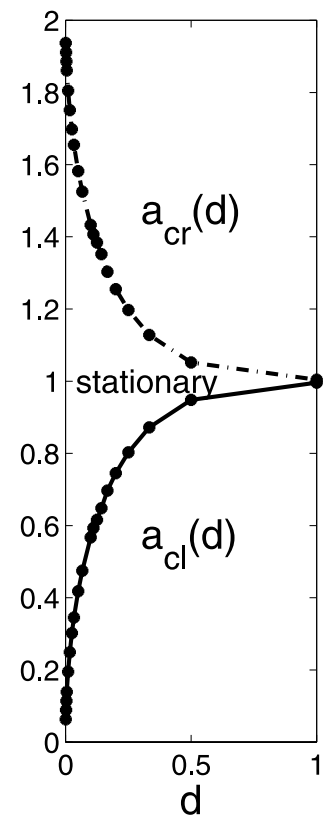

(b)

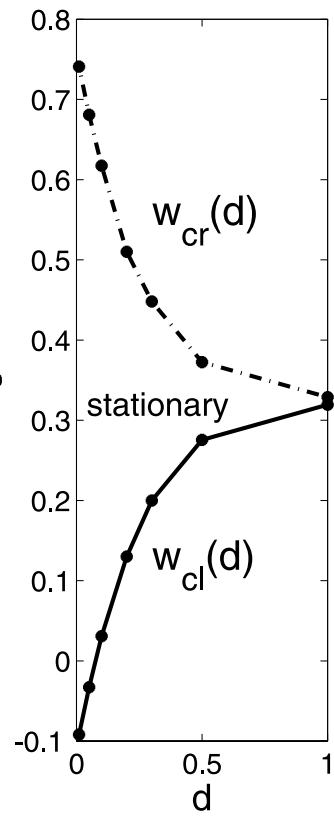

(c)

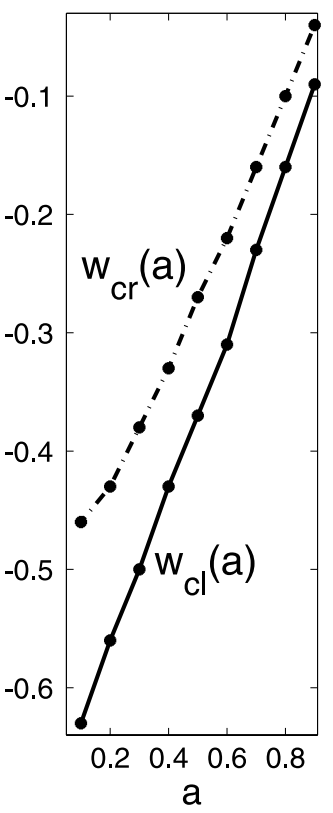

FIG. 1.2. (a) Critical values $a_{c l}$ and $a_{c r}$ as functions of $d$. (b) Critical $w_{c l}(a, d)$ and $w_{c r}(a, d)$ for $a=0.5$. (c) Critical $w_{c l}(a, d)$ and $w_{c r}(a, d)$ for $d=0.5$.

case considered by Booth and Erneux ("slow capture near a limit point"), $d=O\left(a^{2}\right)$, $a \rightarrow 0+$, corresponds to the parameter region in the lowest corner in this figure. In this region, propagation failure is the normal situation. We could, alternatively, fix $a$ and set $f(u)=-w-u(u-a)(u-2)$, using $w$ as the control parameter. Then there are critical values $w_{c l}(a, d)$ and $w_{c r}(a, d)$ such that wave fronts fail to propagate if $w_{c l}<w<w_{c r}$; see Figure 1.2(b). How does the parameter $a$ affect the critical values of $w$ ? Assume that $a<a_{c l}(d)$ for a fixed value of $d$, so that wave fronts propagate for $w=0$. To compensate for this effect, we need a small critical value $w_{c l}(a, d)$ of the parameter $w$. As $a$ departs more and more from $a_{c l}(d)$, larger and larger critical values $w_{c l}(a, d)$ are needed to return to the situation of propagation failure. A similar situation occurs with $w_{c r}(a, d)$. Thus the critical values of $w$ increase (in absolute value) as $\left|a-a_{c}\right|$ increases; see Figure 1.2(c). The effect of increasing the diffusivity $d$ is to shrink the parameter range in which stationary fronts exist. In fact, as $d \rightarrow \infty$ (the continuum limit), the width of the pinning interval is conjectured to decrease exponentially quickly to zero for certain nonlinearities [6, 23]. Propagation failure can be understood as a loss of continuity of the moving front as appropriate critical parameter values are approached. Increasing the discrete diffusivity and deforming the source term sufficiently far from odd symmetry about its middle zero both facilitate the propagation of wave fronts $[7,9]$. Reciprocally, weakening the coupling between cells and diminishing the "external force" $a-1$ helps induce propagation failure.

For the spatially discrete FHN system, the description of wave propagation is more complicated. This also happens for the spatially continuous FHN system $\left(D \partial^{2} u / \partial x^{2}\right.$ instead of discrete diffusion). Depending on the initial condition, stable wave trains or pulses may be approached as time elapses $[27,15,19]$. Pulses cannot be obtained 
for $\epsilon$ larger than a critical value. For discrete diffusion, we can construct pulses, provided that $\epsilon$ is smaller than a critical value $\epsilon_{c}(a, d), a$ is outside a certain interval (corresponding to propagation failure in the scalar case), and the initial condition is chosen appropriately. Our construction combines the theory of front depinning developed in [7] with Keener's asymptotic ideas [19] developed for the FHN model with spatially continuous diffusion. Our results agree very well with direct numerical solutions of (1.2) and (1.3).

The key ideas of an asymptotic theory for (1.2) and (1.3) in the limit as $\epsilon \rightarrow 0$ are simple. First, pulses consist of regions in which $u_{n}(t)$ vary smoothly with $n$, separated by moving sharp interfaces (fronts). In the first type of region, we may set $\epsilon=d=0$ and obtain a description of slow recovery. The sharp interfaces are wave fronts with smooth profiles, $u_{n}(t)=u(z), v_{n}(t)=v(z)$, with $z=n-c t / \epsilon$. Then $v$ is constant at each side of a front, and the excitation variable $u$ obeys the spatially discrete Nagumo equation, whose fronts we can characterize [7,9]. A stable pulse is obtained when the velocity of the leading front is equal to that of the trailing front [19]. This condition fixes the pulse width. Its violation or propagation failure of any of the fronts bounding the pulse result in propagation failure thereof. Notice that we use an analytic expression for the wave front velocity of the Nagumo equation, valid as $a$ is near its critical value for propagation failure. For small values of $d$, the front propagation range is narrow, and the formula for wave front velocity holds for all appropriate values of $a$; see Figure 1.2(a). For larger values of $d$, the interval where propagation occurs is wide, and we can use our approximation only for $a$ close to its critical values $a_{c l}$ and $a_{c r}$. Outside these parameter ranges, the velocity of the Nagumo wave fronts should be calculated numerically.

The rest of the paper is organized as follows. In section 2 , we recall certain needed results on wave front propagation and failure for the spatially discrete scalar reaction-diffusion (Nagumo) equation. Section 3 contains the main theoretical ideas of this paper, with the asymptotic construction of pulses for the discrete FHN system. These ideas and our results are tested by numerically solving the FHN system with appropriate boundary conditions in section 4. Comments on propagation failure of pulses in the FHN system are made in section 5 . Section 6 briefly discusses how a pulse may be generated by applying a temporary stimulus at one end of a fiber with finitely many nodes. The last section contains our conclusions.

2. The spatially discrete Nagumo equation. We consider the equation

$$
\frac{d u_{n}}{d s}=d\left(u_{n+1}-2 u_{n}+u_{n-1}\right)+u_{n}\left(2-u_{n}\right)\left(u_{n}-a\right)-w
$$

for some constant $w$ and denote $h(u, w, a)=u(2-u)(u-a)-w$. As long as $\min h(u, 0, a)<w<\max h(u, a, w)$, this is a "cubic" source having three zeroes $U_{i}(w, a), i=1,2,3, U_{1}<U_{2}<U_{3}$. Wave front solutions joining $U_{1}$ and $U_{3}$ (the two stable zeros) exist. A theory of the pinning and propagation of fronts for this type of equation has been developed in [7,8]. We sketch its implications for (2.1) below.

First assume $w=0$, so that the asymmetry of the source is controlled by the parameter $a$. For $d$ fixed, there are values $a_{c l}(d)$ and $a_{c r}(d)$ such that the following hold:

- The fronts joining $u=0$ and $u=2$ are stationary if $a_{c l}(d) \leq a \leq a_{c r}(d)$. No front propagation is possible.

- Outside this interval, there exist traveling wave fronts $u_{n}(s)=u(n-c s)$ joining 0 and 2. For $a>a_{c r}(d)$, increasing fronts move to the right and de- 
creasing fronts move to the left. For $a<a_{c l}(d)$, fronts move in the opposite way: decreasing fronts move to the right and increasing fronts move to the left. The values $a_{c l}(d)$ and $a_{c r}(d)$ can be approximately calculated as follows. In a large lattice, we decrease or increase $a$ from 1 until we obtain a stationary solution $u_{n}(a)$ whose linear stability problem has a zero eigenvalue; see Figure 1.2.

Now we fix $a$ and vary $w$. The asymmetry of the source is controlled by $a$ and $w$. For fixed $d$ and $a$, critical values $w_{c l}(a, d)$ and $w_{c r}(a, d)$ are found such that the following hold:

- The fronts joining $U_{1}(w, a)$ and $U_{3}(w, a)$ are stationary if $w_{c l}(a, d) \leq w \leq$ $w_{c r}(a, d)$.

- Outside this interval, there exist traveling wave fronts $u_{n}(s)=u(n-c s)$ joining $U_{1}(w, a)$ and $U_{3}(w, a)$. For $w<w_{c l}$, these fronts move to the left if they increase from $U_{1}$ to $U_{3}$, and to the right if they decrease from $U_{3}$ to $U_{1}$. For $w>w_{c r}$, fronts decreasing from $U_{3}$ to $U_{1}$ move to the left, and increasing fronts move to the right.

To calculate $w_{c l}$ and $w_{c r}$, we start by fixing $a$ and finding a value $w=w_{0}$ at which stationary solutions exist for a large lattice. We now decrease or increase $w$ from this value until we obtain a stationary solution $u_{n}(w)$ whose linear stability problem has a zero eigenvalue; see Figure 1.2.

For $w$ near any of its critical values, we can use the following formula to predict the speed of the fronts for $|w|>\left|w_{c}\right|$ :

$$
c(a, d, w) \sim \operatorname{sign}\left(w-w_{c}\right) \frac{\sqrt{\alpha \beta\left(w-w_{c}\right)}}{\pi} .
$$

The parameters $\alpha$ and $\beta$, given by $\alpha=\sum \phi_{n}, \beta=\frac{1}{2} \sum\left[-6 u_{n}\left(w_{c}\right)+2(2+a)\right] \phi_{n}^{3}$ (see $[7,9])$, are functions of $a, d$, and the critical value of $w$. In these formulas, $\phi$ is a positive eigenfunction of the linear stability problem for $u_{n}\left(w_{c}\right)$ with $\sum \phi_{n}^{2}=1$, and $u_{n}\left(w_{c}\right)$ is a stationary solution of (2.1) with $w=w_{c}$ [9]. If $w$ is not close to its critical values, the speed $c(a, d, w)$ should be calculated numerically.

A peculiarity of the Nagumo equation is the scenario for front propagation failure. As we approach the critical values for $a, w$, or any other appropriate pinning control parameter, the front profiles become less smooth and a number of steps appear. In the limit as the control parameter tends to its critical value, the transition regions between steps become infinitely steep, the front profile becomes discontinuous, and its velocity vanishes $[7,9]$.

3. Asymptotic construction of pulses. As we will discuss below, an appropriate initial condition evolves towards a pulse. In particular, we need to fix the parameters $d>0, a<a_{c l}(d)$ (the case $a>a_{c r}(d)$ follows by symmetry), and $\epsilon$ smaller than a certain critical value, $\epsilon_{c}(a, d)$. This last condition also holds for the spatially continuous FHN system, which has two pulse solutions (one stable and one unstable) for $\epsilon<\epsilon_{c}$. These solutions coalesce at $\epsilon_{c}$ and cease to exist for larger $\epsilon$ (see $[26,27]$ ). A pulse consists of regions of smooth variation of $u$ on the time scale $t$, separated by sharp interfaces in which $u$ varies rapidly on the time scale $T=t / \epsilon$. In the regions where $u$ varies smoothly, we can set $\epsilon=d=0$, thereby obtaining the reduced problem

$$
\begin{array}{r}
u_{n}\left(2-u_{n}\right)\left(a-u_{n}\right)-v_{n}=0 \\
\frac{d v_{n}}{d t}=u_{n}-B v_{n} .
\end{array}
$$


These regions are separated by sharp interfaces (moving fronts), at which $u_{n}$ varies rapidly as $u_{n}(t)=u(z), v_{n}(t)=v(z)$, with $z=n-c t / \epsilon$. There, to leading order,

$$
\begin{aligned}
& -c \frac{d u}{d z}=d[u(z+1)-2 u(z)+u(z-1)]+u(z)(2-u(z))(a-u(z))-v \\
& -c \frac{d v}{d z}=0
\end{aligned}
$$

Thus $v$ is a constant equal to the value $v_{n}(t)$ at the last point in the region of smooth variation before the front. Equation (3.3) has a wave front solution as discussed in the previous section. We can now discuss different regions in the asymptotic description of a pulse as follows:

1. The region of smooth variation of $u$ in front of the pulse, described by (3.1) and (3.2). In this region, $u_{n}=U_{1}\left(v_{n}\right)$, so that

$$
\frac{d v_{n}}{d t}=U_{1}\left(v_{n}\right)-B v_{n},
$$

and initial data evolve exponentially fast towards equilibrium, $u_{n}=v_{n}=0$.

2. The pulse leading edge. Let $v(t)$ be the value of $v_{n}$ at the last point of the region in front of the pulse. Eventually, $v \rightarrow 0$. At the leading edge, $u_{n}(t)=u(n-c t / \epsilon)$ is a wave front moving towards the right with speed $C=c(a, d, v) / \epsilon$ measured in points per unit time $t$. We have the boundary conditions $u(-\infty)=U_{3}(v)$ and $u(\infty)=U_{1}(v)$ for the monotone decreasing profile $u(z)$ which satisfies (3.3). It is convenient to call $c_{-}(v)=c(a, d, v)$. Eventually, $C \sim c_{-}(0) / \epsilon$, and $u_{n}$ decreases from $u_{n}=2$ to $u_{n}=0$ across the leading edge of the pulse.

3. The region between fronts: $u_{n}=U_{3}\left(v_{n}\right)$ and

$$
\frac{d v_{n}}{d t}=U_{3}\left(v_{n}\right)-B v_{n} .
$$

There is a finite number of points in this region. On its far right, $v_{n}=v \rightarrow 0$. As we move towards the left, $v_{n}$ increases until it reaches a certain value $V(t)$ corresponding to that in the trailing wave front.

4. The trailing wave front: $v_{n}(t)=v(z)=V$, and $u_{n}(t)=u(z)$ obeys (3.3) with boundary conditions $u(-\infty)=U_{1}(V)$ and $u(\infty)=U_{3}(V)$. This front increases monotonically with $z$, and it moves with speed $C=c(a, d, V) / \epsilon$ measured in points per unit time $t$. It is convenient to denote $c_{+}(V)=$ $c(a, d, V)$. We shall indicate how to determine $V$ below. Clearly, if the pulse is to move rigidly, we should have $c_{+}(V)=c_{-}(0)$ after a sufficiently long transient period.

5. Pulse tail. Again $u_{n}=U_{1}\left(v_{n}\right)$ and $d v_{n} / d t=U_{1}\left(v_{n}\right)-B v_{n}$. Sufficiently far to the left, $v_{n}=u_{n}=0$.

The number of points between wave fronts of the pulse is not arbitrary: it can be calculated following an argument due to Keener for the spatially continuous case [19]. Let $\tau$ be the delay between fronts, i.e., the time elapsed from the instant at which the leading front traverses the point $n=N$ to the instant when the trailing front is at $n=N$. Clearly,

$$
\tau=\int_{v(t-\tau)}^{V(t)} \frac{d v}{U_{3}(v)-B v} .
$$


The number of points between fronts, $l(t)$, can be calculated as

$$
l=\frac{1}{\epsilon} \int_{t-\tau}^{t} c_{-}(v(t)) d t
$$

On the other hand, the separation between fronts satisfies the equation

$$
\frac{d l}{d t}=\frac{c_{-}(v(t))-c_{+}(V(t))}{\epsilon}
$$

The three equations (3.5), (3.6), and (3.7) can be solved to obtain the three unknowns $\tau, l$, and $V(t)$. (The function $v(t)$ is determined by solving (3.2) with $u_{n}=U_{1}\left(v_{n}\right)$ in the region to the left of the leading front.)

After a transient period, $v(t) \rightarrow 0$ and $V(t) \rightarrow V$ (a constant value), so that we have the simpler expressions

$$
\begin{gathered}
\tau=\int_{0}^{V} \frac{d v}{U_{3}(v)-B v}, \\
\frac{d l}{d t}=\frac{c_{-}(0)-c_{+}(V)}{\epsilon},
\end{gathered}
$$

instead of (3.5) and (3.7), respectively. The number of points at the pulse top is now

$$
l=\frac{c_{-}(0) \tau}{\epsilon}=\frac{c_{-}(0)}{\epsilon} \int_{0}^{V} \frac{d v}{U_{3}(v)-B v} .
$$

This equation yields $V$ as a function of $l$. Then (3.9) becomes an autonomous differential equation for $l$ that has a stable constant solution at $l=l^{*}$ such that $c_{-}(0)=c_{+}(V(l))$ : at $l=l^{*}$, the right-hand side of (3.9) has a slope $-\left[U_{3}(V)-\right.$ $B V] c_{+}^{\prime}(V) / c_{-}(0)<0$.

Recapitulating, for appropriate initial conditions, the leading and trailing fronts of a pulse evolve until $l$ reaches its stable value at which $c_{-}(0)=c_{+}\left(V\left(l^{*}\right)\right)$ and $(3.10)$ holds. To compute $l^{*}$, we first determine $V^{*}=V\left(l^{*}\right)$ by using $c_{-}(0)=c_{+}\left(V\left(l^{*}\right)\right)$. Then we calculate $\tau=\tau^{*}$ (which does not depend on $\epsilon$ !) from (3.8) and $l^{*}=c_{-}(0) \tau^{*} / \epsilon$. Our construction breaks down if the number of points between fronts falls below 1 . This yields an upper bound for the critical value of $\epsilon$ above which pulse propagation fails: $\epsilon_{c} \sim c_{-}(0) \tau^{*}$.

The asymptotic length of the pulse tail is obtained by first calculating the time needed for $v_{n}$ to go from 0 to $V\left(l^{*}\right)$ to the left of the trailing front: $T=\int_{0}^{V} d v /\left[U_{1}(v)-\right.$ $v]$. The tail length is then $L=c_{-}(0) T / \epsilon$.

4. Numerically calculated pulses. We shall compare numerical solutions for different representative values of $d$ with the approximate pulses provided by our theory. As initial data, we have adopted our approximate pulses. We have also used humplike profiles with compact support for $u_{n}(0)$ and $v_{n}(0)$. It is important that $v_{n}(0)=0$ at the leading edge of $u_{n}(0)$ and to its right, and that $v_{n}(0)=V \approx w_{c r}(d)$ at the trailing edge, where $v_{n}(0)$ reaches its maximum. Had we chosen $v_{n}(0)=0$ for all $n$, the $u_{n}$ profile would have split into two pulses traveling in opposite directions as time elapsed; see Figure 4.1. The region between leading and trailing fronts of the pulse acquires its asymptotic shape quite quickly, but the pulse tail is usually rather long and evolves slowly towards its final form. 

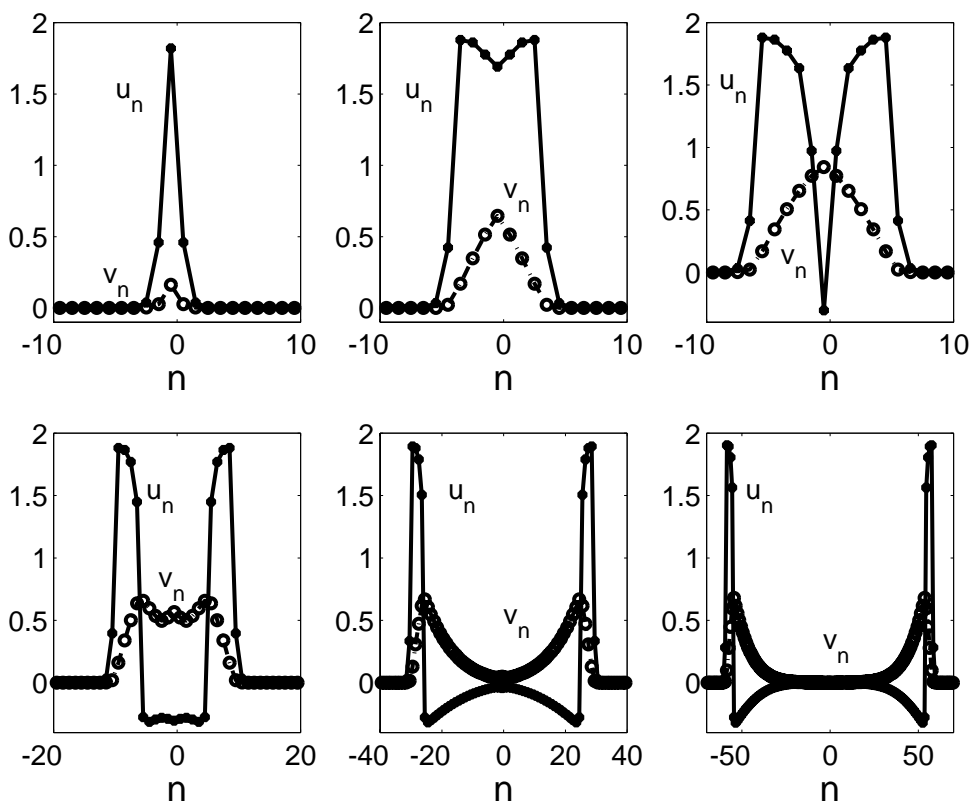

FIG. 4.1. Splitting of an initial profile into two pulses propagating in opposite directions for $d=0.1, a=0.5$, and $\epsilon=0.006$.

- $d=1$. In this case, $a_{c l}=0.996$. We choose $a=0.99<a_{c l}$ and analyze front propagation for the rescaled Nagumo equation (2.1) first. The front propagation thresholds for these values of $d$ and $a$ are $w_{c l}=0.0038$ and $w_{c r}=0.0095$. Figure $4.2(\mathrm{a})$ shows the speeds of leading and trailing fronts as functions of $w$, as predicted by (2.2). For $w=0$, the leading front should move at speed $c_{-}(0)=0.0093$. The relation $c_{+}(V)=c_{-}(0)$ yields the asymptotic value $V^{*}=0.0133$ at the trailing front joining $U_{1}\left(V^{*}\right)=-0.00665$ to $U_{3}\left(V^{*}\right)=1.9933$. The time elapsed between fronts is $\tau^{*}=0.00652$, as calculated from (3.8). Then our upper bound for the critical value of $\epsilon$ is $\epsilon_{c}=0.000064$. Choosing a smaller value, $\epsilon=0.000005$, we obtain a pulse speed of $C=c_{-}(0) / \epsilon=1869$ points per unit time and a pulse width of $l^{*}=C \tau^{*} \sim 13$ points. Our numerical solution of the full FHN system (1.2) and (1.3) yields a pulse speed $C=2000$ and a width of 13 points for $\epsilon=0.000005$. The trailing front joins -0.006647 and 1.993 with $V^{*}=-0.0133$; see Figure 4.2(b). Note that the relative error in the predicted speed $C$ is 0.0655 . Obviously, rescaling the speed to $C=c_{-}(0) / \epsilon$ amplifies the error in our predictions. We have not been able to observe pulses for $\epsilon \geq 0.0000076$, which is smaller but not far from our estimation $\epsilon_{c}=0.000064$. Let us now choose $a=0.5$ which is far from $a_{c l}$. Then $w_{c l}=0.3194$ and $w_{c r}=0.3287$. Equation $(2.2)$ predicts $c_{-}(0)=0.09983$, whereas the trailing front joins -0.316 to 1.71 at $V^{*}=0.6$. If $\epsilon=0.01$, the speed and width of the pulse are $C=9.983$ and $l^{*}=0.351 C \sim 4$, according to our theory. Numerically, we observe $C=64.7$ and $l^{*}=25$. The source of these large errors is the value $c_{-}(0)=0.09983$ predicted with formula (2.2). If we replace this value by the numerical front speed calculated directly, $c_{-}(0)=0.673$, we obtain $C=67.3$ and $l^{*} \sim 24$ points, which better 


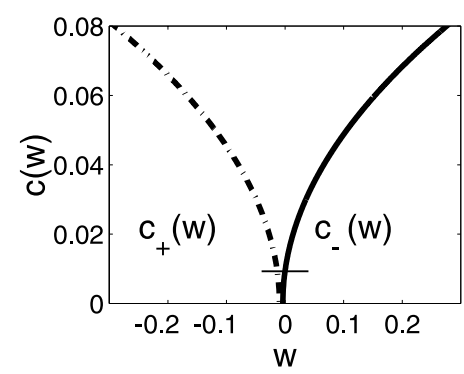

(a)
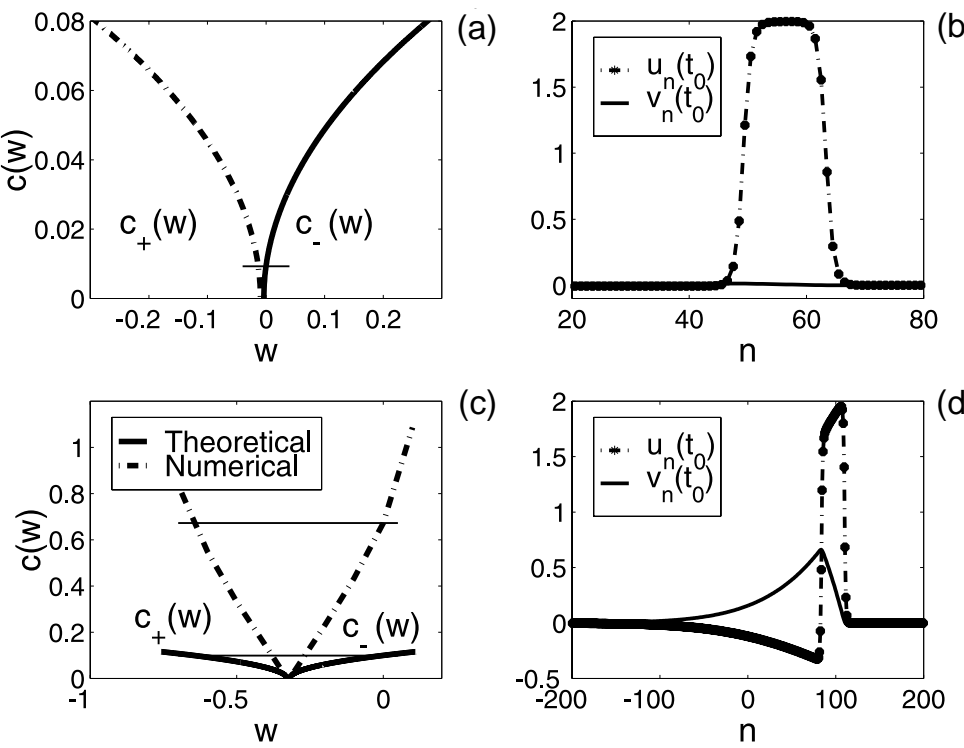

(c)

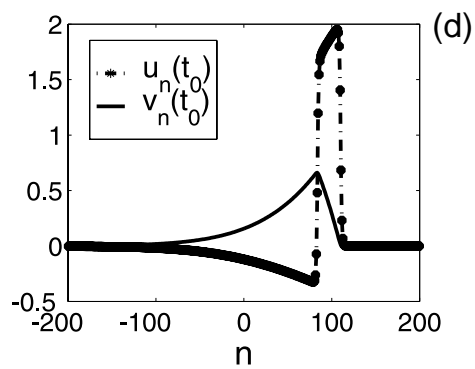

FIG. 4.2. (a) Predicted speeds for wave fronts of the Nagumo equation (2.1) with $d=1, a=0.99$. The horizontal line marks the condition $c_{+}(w)=c_{-}(0)$, thereby graphically yielding $w=V^{*}$. (b) FHN pulse for $\epsilon=0.000005$. (c) Predicted and numerical speeds for wave fronts of (2.1) with $d=1$, $a=0.5$. The horizontal lines mark $c_{+}(w)=c_{-}(0)$. (d) FHN pulse for $\epsilon=0.01$.

fit the numerically observed values.

- $d=0.1$. In this case, $a_{c l}=0.567$, and we shall choose $a=0.5<a_{c l}$. Let us first analyze front propagation for the rescaled Nagumo equation (2.1). For these values of $d$ and $a$, we obtain $w_{c l}=0.0307$ and $w_{c r}=0.6175$. Figure 4.3(a) shows the predicted speeds of the leading and trailing fronts as functions of $v$, as given by formula (2.2). For $w=0$, the leading front should move with speed $c_{-}(0)=0.075662$. At the trailing front, $c_{+}(V)=c_{-}(0)$ yields $V^{*}=0.648, U_{1}\left(V^{*}\right)=-0.33328$, and $U_{3}\left(V^{*}\right)=1.666$. The time elapsed between fronts is $\tau^{*}=0.39266$, which gives $l^{*}=0.0297 / \epsilon$. Our bound for the critical value of $\epsilon$ is $\epsilon_{c}=c_{-}(0) \tau^{*}=0.029$. Selecting $\epsilon=0.003$, we predict $C=25.22$ and $l^{*} \sim 10$ points. Direct numerical calculations yield a pulse speed $C=26.38$ and a pulse width of about 10 points. The trailing front joins -0.3269 to 1.675 with $V^{*}=0.6578$; see Figure 4.3(b). We have not been able to obtain pulses for $\epsilon \geq 0.007$, which is four times smaller than our upper bound of 0.029 .

- $d=0.01$. In this case, $a_{c l}=0.195$, and we shall choose $a=0.1<a_{c l}$. Let us first analyze front propagation for the rescaled Nagumo equation (2.1). The front propagation thresholds for these values of $d$ and $a$ are $w_{c l}=0.0136$ and $w_{c r}=1.0784$. Figure 4.4(a) shows the predicted speeds of leading and trailing fronts as functions of $w$ according to (2.2). For $w=0$, the leading front should move with speed $c_{-}(0)=0.052$. Then the trailing front has $V^{*}=1.092$ corresponding to $c_{+}(V)=c_{-}(0)$, and it joins $U_{1}\left(V^{*}\right)=-0.6$ to $U_{3}\left(V^{*}\right)=1.4$. The time elapsed between fronts is $\tau^{*}=0.748$, and the pulse width, $l^{*}=0.297 / \epsilon$. Our bound for the critical value of $\epsilon$ is $\epsilon_{c}=$ $c_{-}(0) \tau^{*}=0.058$. Selecting $\epsilon=0.001$, we predict $C=52$ and $l^{*}=39$ points. 

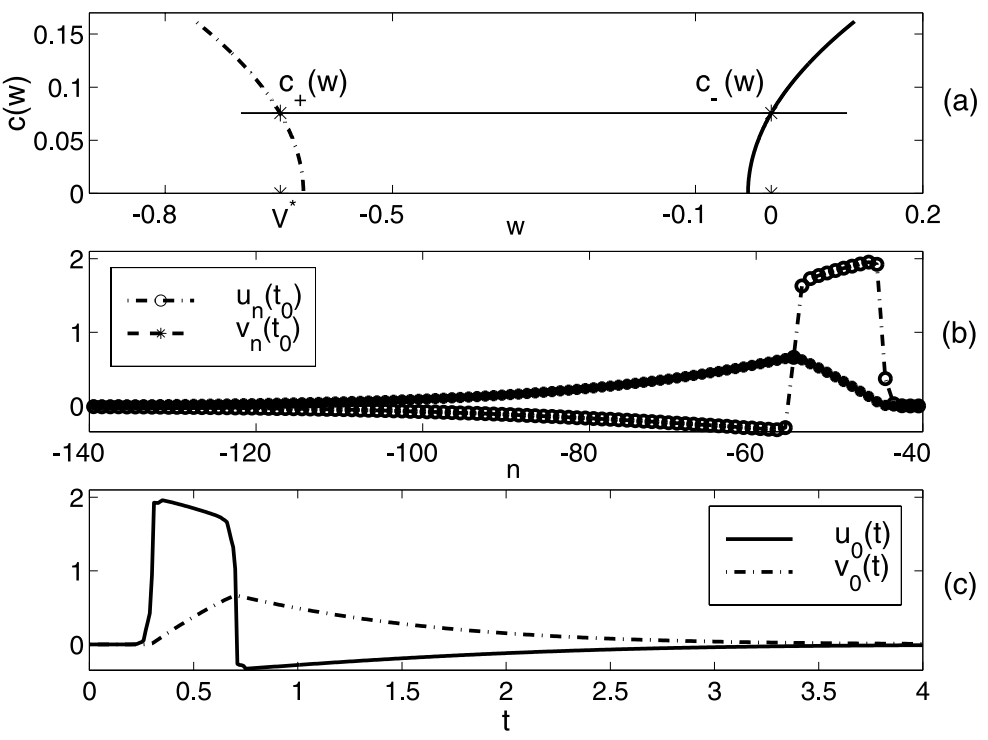

FIG. 4.3. (a) Predicted speeds for the Nagumo equation (2.1) with $d=0.1$ and $a=0.5$. The horizontal line graphically yields $V^{*}$ such that $c_{+}\left(V^{*}\right)=c_{-}(0)$. (b) Profiles of the FHN pulse for $\epsilon=0.003$ (c) Trajectories of one point, $u_{0}(t), v_{0}(t)$, as the FHN pulse propagates through it.

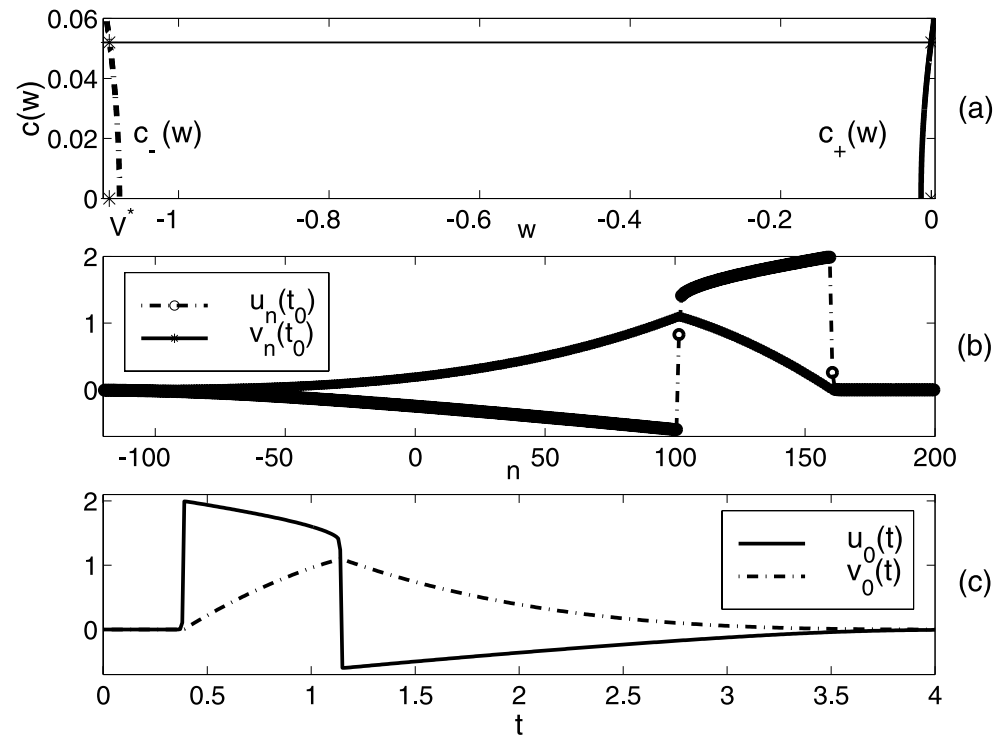

FIG. 4.4. (a) Predicted speeds for the Nagumo equation (2.1) with $d=0.01$ and $a=0.1$. (b) FHN pulse for $\epsilon=0.001$. (c) Trajectory of one point, $u_{0}(t)$, as the FHN pulse propagates through it. 
Numerical observations yield $C=77.7$ (a relative error of 0.3 ) and a pulse width of 59 points. Furthermore, the trailing front joins -0.59 to 1.4 with $V^{*}=1.095$; see Figure 4.4(b). Again the observed errors in the pulse speed and width are due to errors in the prediction of $c_{-}(0)$ given by formula (2.2). Replacing this value by the numerically computed front speed $c_{+}(0)=0.078$, we obtain $C=78$ and $l^{*} \sim 58$, better fit to the real values.

Let us now describe the situation for other values of $d$. Our asymptotic theory agrees with the numerical results, provided that $\epsilon$ is sufficiently small, but the velocity of the Nagumo wave fronts should be either approximated by (2.2) or calculated numerically depending on how close to zero $w_{c l}$ happens to be. For $d<0.01$, the length of the intervals in which fronts of the Nagumo equation propagate is very small. Then the front speeds are always very small and given by (2.2) with great accuracy. Our asymptotic description of the pulse agrees very well with numerical solutions of the FHN system. If $d>1$, the spatially discrete FHN system can be approximated by its continuum limit. The length of the pinning intervals for the Nagumo equation is below 0.001, and the wave front velocities are essentially a correction of the wave front velocities for the spatially continuous Nagumo equation (see [21]):

$$
\begin{aligned}
c & =\sqrt{d} c_{0}\left(1-\frac{k_{1} c_{0}^{2}}{2 d}+O\left(\frac{c_{0}^{4}}{d^{2}}\right)\right), \\
c_{0} & =\frac{2 U_{2}(w)-U_{1}(w)-U_{3}(w)}{\sqrt{2}}, \\
k_{1} & =-\frac{\int_{-\infty}^{\infty} e^{-c_{0}^{2} s} V_{0}^{\prime}(s) V_{0}^{\prime \prime \prime \prime}(s) d s}{12 c_{0}^{4} \int_{-\infty}^{\infty} e^{-c_{0}^{2} s} V_{0}^{\prime}(s) V_{0}^{\prime \prime}(s) d s} .
\end{aligned}
$$

Here $V_{0}$ is the appropriate wave front solution of the equation

$$
c_{0}^{-2} V_{0}^{\prime \prime}-V_{0}^{\prime}+V_{0}\left(2-V_{0}\right)\left(V_{0}-a\right)-w=0 .
$$

5. Propagation failure. Two facts may lead to propagation failure: a value of $\epsilon$ that is too large or $a \in\left(a_{c l}(d), a_{c r}(d)\right)$.

Let us consider the first cause of propagation failure now. If $\epsilon$ surpasses a certain critical value $\epsilon_{c}$, recovery is too fast and a stable pulse cannot be sustained. This situation also occurs in spatially continuous FHN systems. In these systems, there exist two pulses (one pulse is stable, the other unstable) for $\epsilon<\epsilon_{c}$; they coalesce at $\epsilon_{c}$ and cease to exist for larger $\epsilon$. In the discrete FHN system, the phenomenon of wave front propagation failure implies that pulses may propagate only if $a<a_{c l}(d)$ or $a>a_{c r}(d)$. As indicated by (3.10), the number of points between the two fronts of the stable pulse decreases as $\epsilon$ increases towards $\epsilon_{c}(a, d)$. Eventually the two fronts coalesce, and it is not possible to propagate a stable pulse for $\epsilon>\epsilon_{c}(a, d)$. If we start with an appropriate pulse-like initial condition, we find the scenario of propagation failure depicted in Figures 5.1 and 5.2. For small $d(d=0.1)$, the variable $v_{n}$ ceases to be almost constant at the leading edge of the pulse, and the distance between the two fronts diminishes. While $v_{n} \sim 0$ at the rightmost point of the leading front, $v_{n} \sim w>0$ at the leftmost point. Thus, $u_{n}$ in this front decreases from $U_{3}(w)$ to zero as $n$ increases. The value $w$ increases with $\epsilon$, and $U_{3}(w)$ decreases. At the same time, the leading front speed diminishes as $w$ increases until $w$ surpasses the propagation threshold and the leading front stops. Since the back front goes on moving, the pulse vanishes; see Figure 5.1. For large $d(d=1)$, a decremental pulse is formed. Its 

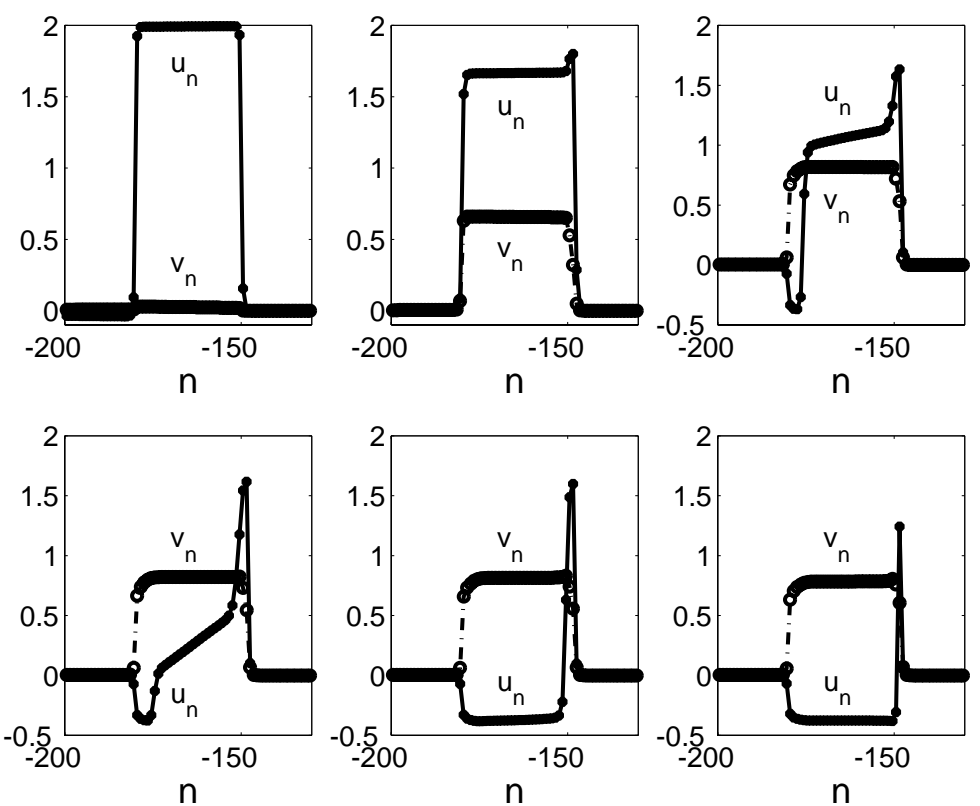

FIG. 5.1. Snapshots of the excitation and recovery variables for $d=0.1, a=0.5$, and $\epsilon=0.007$, illustrating propagation failure of the pulse for $\epsilon>\epsilon_{c}$.
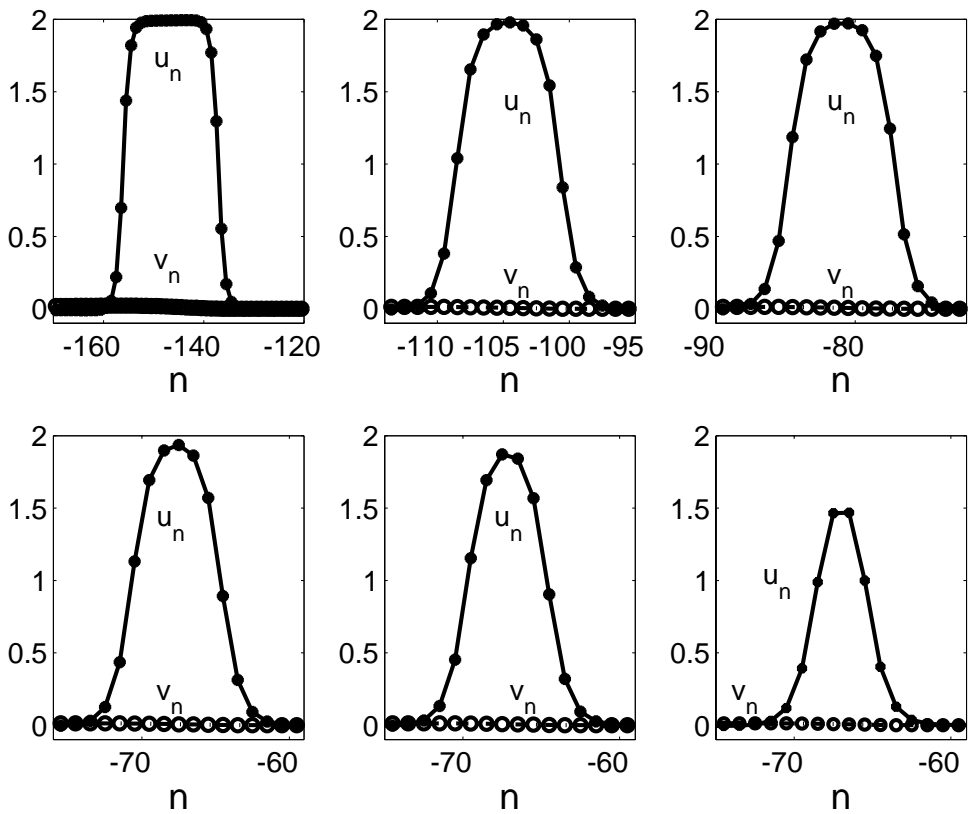

FIG. 5.2. Same as Figure 5.1 for $d=1, a=0.99$, and $\epsilon=0.0000076$. 
width and height decrease as it moves until it disappears; see Figure 5.2. Numerical simulations of the FHN system show that $\epsilon_{c} \rightarrow 0$ as $a$ tends to either $a_{c l}(d)$ or $a_{c r}(d)$.

Let us assume now that $a \in\left(a_{c l}(d), a_{c r}(d)\right)$. Then the leading front cannot propagate with $v_{n}=v=0$. We need $v_{n} \sim v<w_{c l}(a, d)<0$. However, in the region in front of the leading edge, $v_{n}$ and $u_{n}$ evolve towards 0 , whereas we have $u_{n}>0$ at the leading front. Thus $d v_{n} / d t=u_{n}-B v_{n} \geq 0$ there, and $v_{n}$ will increase until $v_{n}>0$, which contradicts our previous assumption. Thus we cannot have stable propagating pulses. Furthermore, there are no stationary pulses of the type we have discussed for this range of $a$ : if $v_{n}=u_{n} / B$, the source $u_{n}\left(2-u_{n}\right)\left(a-u_{n}\right)-v_{n}=$ $u_{n}\left(2-u_{n}\right)\left(u_{n}-a\right)-u_{n} / B$ has only one zero, not three as in our construction. This does not preclude the existence of other pulses, such as those corresponding to the homoclinic orbit in the phase space of the spatially continuous FHN system. However, we have not observed stable stationary pulses of this type in the spatially discrete FHN system.

6. Pulse generation at a boundary. So far, we have considered the motion of a pulse (or its failure) in a sufficiently large myelinated nerve fiber. We have not discussed how such a pulse might be created in a more realistic situation. Clearly, nerve fibers have finitely many nodes of Ranvier, and pulses are typically generated at the fiber boundary. Thus we are led to consider how a pulse might be generated by an excitation at a boundary and how the pulse propagates or fails in a finite fiber. This problem was tackled by Booth and Erneux [5] using parameter values for which the FHN pulse fails to propagate. We shall now discuss different parameter ranges.

Nerve fibers may have either a few nodes of Ranvier (e.g., 20 for neurons of the central nervous system [25]) or several hundred nodes (in the peripheral nervous system [29]). Thus we shall consider a finite FHN system with $N$ nodes and a Neumann boundary condition at the right end, $u_{N+1}=u_{N}$. At the left end, we impose $u_{0}(t)=2$ for $0 \leq t \leq 0.05$, and $u_{0}(t)=0$ for $t>0.05$. The results corresponding to parameter values $d=0.1$ and $a=0.5$ are depicted in Figures 6.1 (for which $\epsilon=0.006$ ) and 6.2 (for which $\epsilon=0.003$ ). The asymptotic theory predicts that fully developed FHN pulses (corresponding to $N=\infty$ ) would have widths of $l^{*} \approx 5$ and $l^{*} \approx 10$, respectively. The left boundary condition ensures that the membrane potential $u_{n}$ is excited during sufficient time, so that a wave is generated at the left end of the fiber.

The excitation at the left boundary induces a wave front that propagates with a velocity given approximately by $C=c_{-}(0) / \epsilon$ along the finite fiber for the parameter values we consider. For example, $C \approx 12.6$ for $\epsilon=0.006$, which is close to the numerically observed value of 10 in Figure 6.1. Similarly, $C \approx 25.22$ for $\epsilon=0.003$, which is close to the numerically observed value of 26 in Figure 6.2. If the fiber is long enough, a second wave front follows the first one, and their mutual distance rapidly approaches the asymptotic value $l^{*}$. (The number of nodes between fronts is 4 in Figure 6.1, while the asymptotic theory predicts $l^{*} \approx 5$; in Figure 6.2, numerical observation confirms the asymptotic value $l^{*} \approx 10$.) The numerical solution of the finite FHN system shows that an eventually truncated FHN pulse comprising the two wave fronts and the region between them is formed, provided that $N$ is at least twice $l^{*}$. Otherwise, at best only the first wave front is shed at the boundary, as shown in Figure 6.2(a). Pulses fail to propagate in fibers whose parameters fall in the propagation failure region, as discussed in section 5 . 

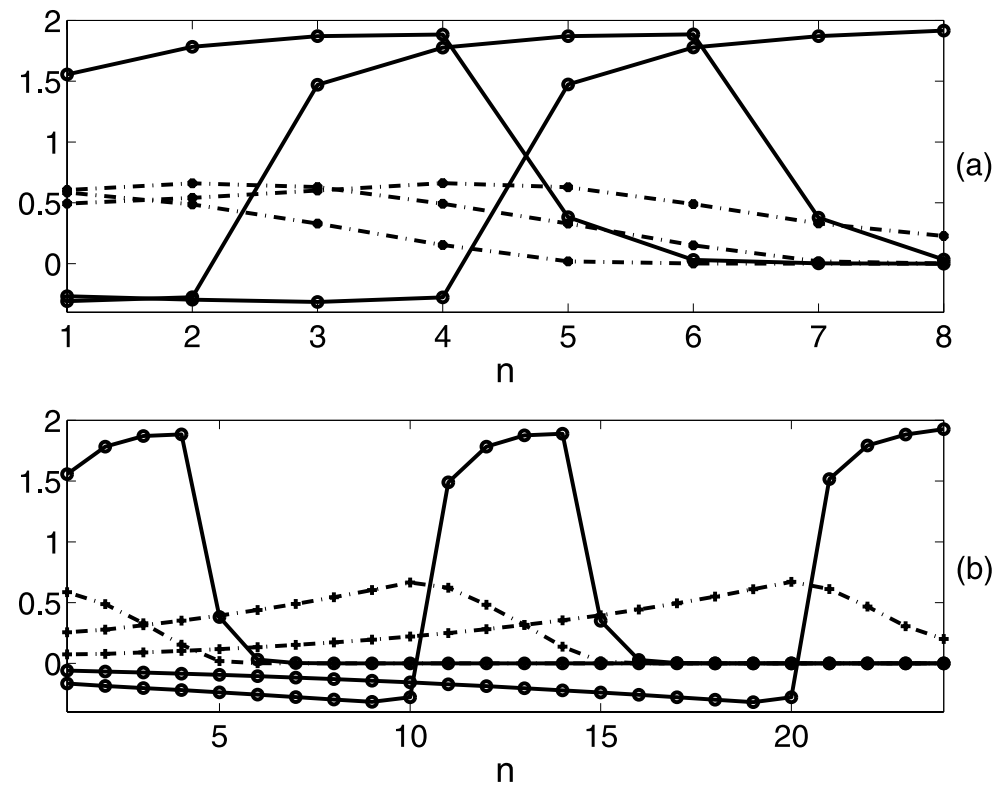

FIG. 6.1. Snapshots of the excitation (solid line) and recovery (dotted line) variables for an FNH system with $N$ nodes and $d=0.1, a=0.5$, and $\epsilon=0.006$. (a) Profiles at times $0.4,0.6$, and 0.8 for $N=8$. (b) Profiles at times $0.4,1.4$, and 2.4 for $N=24$.
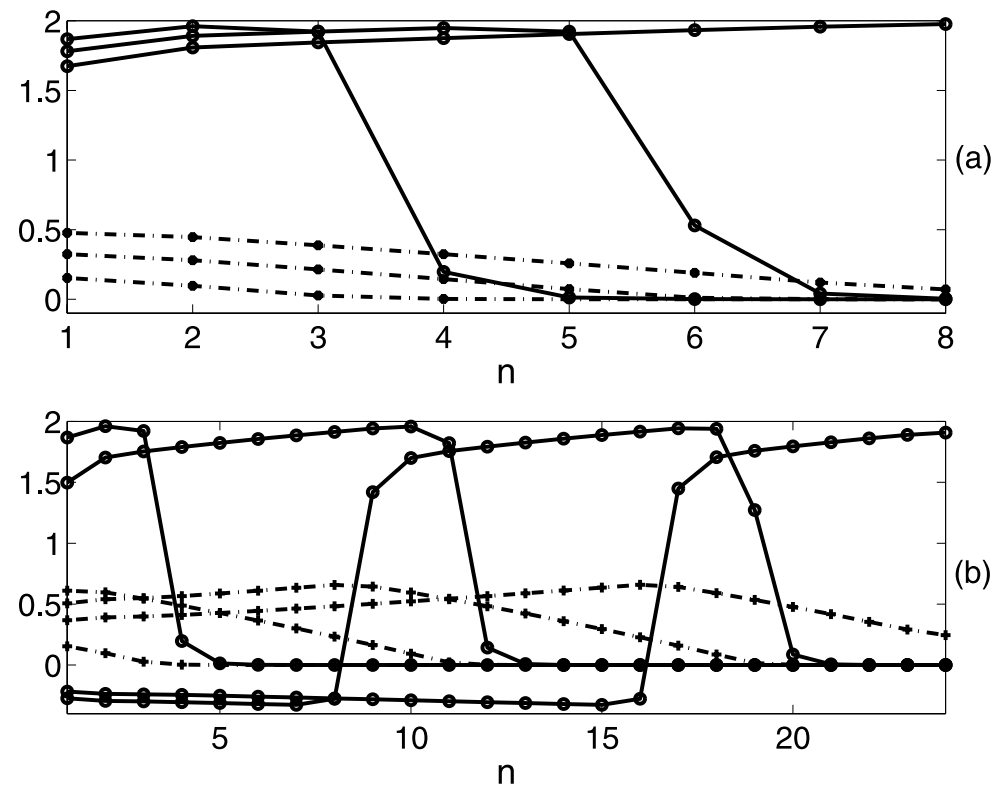

FIG. 6.2. Snapshots of the excitation (solid line) and recovery (dotted line) variables for an FNH system with $N$ nodes and $d=0.1, a=0.5$, and $\epsilon=0.003$. (a) Profiles at times $0.1,0.2$, and 0.3 for $N=8$. (b) Profiles at times $0.1,0.4,0.7$, and 1.0 for $N=24$. 
7. Conclusions. We have constructed stable pulses of the spatially discrete FHN system by asymptotic methods. In a pulse, there are regions where the excitation variable varies smoothly, separated by sharp fronts. These fronts are solutions of the discrete Nagumo equation with a constant value of the recovery variable. Their shape and speed can be approximately calculated near parameter values corresponding to front propagation failure or near the continuum limit. For long times, their width is given by the only stable solution of a one-dimensional autonomous system. We have compared the asymptotic results with numerical solutions of the FHN system and analyzed different scenarios for failure of pulse propagation. Besides the classical scenario of small separation between the time scales of excitation and recovery (large $\epsilon$ as in the spatially continuous FHN system), propagation failure of fronts for the spatially discrete Nagumo equation provides a different mechanism of propagation failure of pulses for the discrete FHN system. Wave fronts and pulses can be generated at a boundary and propagate or fail to propagate along a finite FHN system. If the number of nodes is sufficiently large, the two wave fronts comprising an FHN pulse can be shed at the boundary, and their separation rapidly reaches the value given by the asymptotic theory. This is true even if the fiber is too short to accommodate the slowly varying regions at the back of the second wave front of the pulse. In long fibers, a fully developed FNH pulse may be generated by an over-threshold stimulus applied during a short time at one end of the fiber.

\section{REFERENCES}

[1] A. Amann, A. Wacker, L. L. Bonilla, and E. Schöll, Dynamic scenarios of multistable switching in semiconductor superlattices, Phys. Rev. E (3), 63 (2001), pp. 1-8.

[2] A. R. A. Anderson And B. D. Sleeman, Wave front propagation and its failure in coupled systems of discrete bistable cells modelled by FitzHugh-Nagumo dynamics, Internat. J. Bifur. Chaos Appl. Sci. Engrg., 5 (1995), pp. 63-74.

[3] J. Bell AND C. Costner, Threshold behavior and propagation for nonlinear differentialdifference systems motivated by modeling myelinated axons, Quart. Appl. Math., 42 (1984), pp. $1-13$.

[4] L. L. Bonilla, Theory of nonlinear charge transport, wave propagation and self-oscillations in semiconductor superlattices, J. Phys. Condensed Matter, 14 (2002), pp. R341-R381.

[5] V. Booth AND T. ERneux, Understanding propagation failure as a slow capture near a limit point, SIAM J. Appl. Math., 55 (1995), pp. 1372-1389.

[6] J. W. CAHN, Theory of crystal growth and interface motion in crystalline materials, Acta Metallurgica, 8 (1960), pp. 554-562.

[7] A. Carpio and L. L. Bonilla, Wave front depinning transitions in discrete one-dimensional reaction diffusion equations, Phys. Rev. Lett., 86 (2001), pp. 6034-6037.

[8] A. Carpio, L. L. Bonilla, and G. Dell'Acqua, Wave front motion in semiconductor superlattices, Phys. Rev. E (3), 64 (2001), pp. 1-9.

[9] A. Carpio And L. L. Bonilla, Depinning transitions in discrete reaction-diffusion equations, SIAM J. Appl. Math., to appear.

[10] A. Carpio, S. J. Chapman, S. P. Hastings, and J. B. Mcleod, Wave solutions for a discrete reaction-diffusion equation, European J. Appl. Math., 11 (2000), pp. 399-412.

[11] G. FÁth, Propagation failure of traveling waves in a discrete bistable medium, Phys. D, 116 (1998), pp. 176-190.

[12] R. FitzHugh, Impulse and physiological states in models of nerve membrane, Biophys. J., 1 (1961), pp. 445-466.

[13] J. Frenkel and T. Kontorova, On the theory of plastic deformation and twinning, J. Phys. USSR, 13 (1938), pp. 1-10.

[14] G. GRüneR, The dynamics of charge-density waves, Rev. Modern Phys., 60 (1988), pp. 11291181.

[15] S. P. Hastings, The existence of homoclinic and periodic orbits for FitzHugh-Nagumo's equations, Q. J. Math., 27 (1976), pp. 123-134.

[16] S. P. Hastings And X. Chen, Pulse waves for a semi-discrete Morris-Lecar-type model, J. 
Math. Biol., 38 (1999), pp. 1-20.

[17] R. Hobart, Peierls-barrier minima, J. Appl. Phys., 36 (1965), pp. 1948-1952.

[18] J. Kastrup, R. Hey, K. Ploog, H. T. Grahn, L. L. Bonilla, M. Kindelan, M. Moscoso, A. WaCKer, AND J. Galán, Electrically tunable GHz oscillations in doped GaAs-AlAs superlattices, Phys. Rev. B (3), 55 (1997), pp. 2476-2488.

[19] J. P. KeEner, Waves in excitable media, SIAM J. Appl. Math., 39 (1980), pp. 528-548.

[20] J. P. KeEner, Propagation and its failure in coupled systems of discrete excitable cells, SIAM J. Appl. Math., 47 (1987), pp. 556-572.

[21] J. P. Keener and J. Sneyd, Mathematical Physiology, Springer, New York, 1998, Chapter 9.

[22] J. P. KeEner, Propagation of waves in an excitable medium with discrete release sites, SIAM J. Appl. Math., 61 (2000), pp. 317-334.

[23] J. R. King And S. J. Chapman, Asymptotics beyond all orders and Stokes lines in nonlinear differential-difference equations, European J. Appl. Math., 12 (2001), pp. 433-463.

[24] J. MALLET-PARET, The global structure of traveling waves in spatially discrete dynamical systems, J. Dynam. Differential Equations, 11 (1999), pp. 49-127.

[25] C. C. McIntyre ANd W. M. Grill, Excitation of central nervous system neurons by nonuniform electric fields, Biophys. J., 76 (1999), pp. 878-888.

[26] J. Nagumo, S. Arimoto, and S. Yoshizawa, An active pulse transmission line simulating nerve axon, Proc. Inst. Radio Engineers, 50 (1962), pp. 2061-2070.

[27] J. Rinzel And J. B. KelleR, Traveling wave solutions of a nerve conduction equation, Biophys. J., 13 (1973), pp. 1313-1337.

[28] A. C. Scotт, The electrophysics of a nerve fiber, Rev. Modern Phys., 47 (1975), pp. 487-533.

[29] J. J. Struijk, The extracellular potential of a myelinated nerve fiber in an unbounded medium and in nerve cuff models, Biophys. J., 72 (1997), pp. 2457-2469.

[30] G. de Vries, A. Sherman, And H.-R. Zhu, Diffusively coupled bursters: Effects of cell heterogeneity, Bull. Math. Biol., 60 (1998), pp. 1167-1200.

[31] B. ZinNer, Existence of traveling wavefront solutions for the discrete Nagumo equation, J. Differential Equations, 96 (1992), pp. 1-27. 